\title{
A Numerical Study of the Spread of Malaria Disease with Self and Cross-Diffusion in the System
}

\author{
Bushra Hasan ${ }^{\mathrm{a} ; \mathrm{b}}$, Manmohan Singh ${ }^{\mathrm{a}}$ and David Richards ${ }^{\mathrm{a}}$ \\ ${ }^{a}$ Mathematics Department, Faculty of Science Engineering and Technology, Swinburne University of \\ Technology, Hawthorn, Victoria 3122, Australia. \\ ${ }^{b}$ Diyala University Engineering College, Baaquba, Diyala, Iraq.
}

\begin{abstract}
A study of the SIS model of malaria disease with a view to observing the effects of self and crossdiffusion on spatial dynamics is undertaken. Three different cases based on self-diffusion and cross-diffusion are chosen for the investigation. Two cases of cross-diffusion without self-diffusion are also considered in order to see the effects of diffusion on the transmission of malaria. Basic reproductive numbers and bifurcation values are calculated for each case. A series of numerical simulations based on self and cross-diffusion is performed. It is observed that with positive cross-diffusion and self-diffusion in the system, there is a significant increase in the proportion of both infected human and mosquito populations. The proportion of infected humans increases markedly with cross diffusion in the system. This also gives rise to some oscillations across the domain.
\end{abstract}

Keywords: Self and Cross-Diffusion, Disease-free equilibrium state, Endemic equilibrium state, Basic reproduction number, Numerical simulations, Bifurcation Values.

\section{Introduction}

Malaria is one of the serious infectious diseases incubated by the Plasmodium parasite and transmitted among the human population through female Anopheles mosquitoes. It still remains as one of the most common and deadly human diseases [1]. It is estimated that $45 \%$ of the world's population lives in malaria- endemic areas. The majority of the casualties are children under five years old. Pregnant Women are also quite susceptible to this disease. Most infectious cases resulting in mortality occur in sub-Saharan Africa. It has been estimated that $80 \%$ of infectious and $90 \%$ of fatal cases occur in Africa [2]. The main factors for malaria to be endemic are the environmental conditions prevailing in countries with tropical climates that allow mosquitoes and malaria parasites to reproduce rapidly. For instance, the high moisture, moderate-to-warm temperatures and water catchments are responsible for the spread of malaria. The disease can lead to severe complications involving cerebrum, kidneys, lungs and other organs of the human body. Clinical side effects, such as fever, chills, vomiting, muscular pains, heavy headache and sweats may occur within a few days of a bite from an infected mosquito [34].

Malaria infections by debilitate of the immune system, thus rendering people more susceptible to becoming infected with HIV[6]. In non-stable malaria regions, malaria disease increases mortality in advanced HIV patients by $25 \%$ [13]. Malaria also increases morbidity rates. It continues to be a great global health and socio-economic problem. In particular, it places great burdens on developing countries. It is noteworthy that poverty is a major factor responsible for the spread of Malaria. For instance, populations in poor communities may have bad sanitation and inadequate and such conditions permit mosquitoes to reproduce in large numbers. Being poor, people are not able to bear the cost of a mosquito net or even protective screens for the windows of their houses. A dark clammy room is a favorite hiding place for mosquitoes. An increase in the number of vectors living within the human population leads to an increase in bites by infected mosquitoes. This is how infected Anopheles mosquitoes infect populations from poor backgrounds with the malaria parasite[29].

Malaria disease is considered a global problem, and many scientists, researchers and other epidemiologists have worked on discovering the dynamics and transmission of malaria. Mathematicians have made considerable progress through mathematical modelling giving an understanding into the interaction between the human (host) and mosquitoes (vector) populations. This has led to greater knowledge of the dynamics of malaria and how to combat it. The first mathematical model for malaria transmission was introduced by Sir Ronald Ross in 1916 [30]. This model was extended and modified as an ODE model by Mc Donald [21, 22]. ODE models with acquired immunity were studied later by Dietz [10], Bailey [5] and Aron [4]. Probabilistic models and further developed models were studied by Doolan et al [11] and Ngwa et al [3].

It is usually considered that populations distribute non-homogeneously and thus develop movement strategies. The diffusion processes can possibly define various intensity levels of the population size which can further give rise to different levels of movements. Such movements can be described by considering the concentration of a species or group alone ( passive or selfdiffusion) and in relation to those of other species or groups (cross-diffusion). In general, endemic models that include self and cross-diffusion can enable better understanding of population dynamics [14]. Self-diffusion is the term for the movement of people from higher to lower concentration region. Cross-diffusion refers to the flux population of one species or group due to the presence of other species or groups. The study of self and cross-diffusion has gained considerable interest in the field of population dynamics, mainly due to their ability to predict some important characteristics of the spatial distribution of species populations [19]. 
The phenomenon of cross-diffusion diffusion in nature can be characterised as the propensity of the susceptible to avoid the infected [33]. Cross-diffusion expresses the population flux of a given subpopulation affected by the presence of other subpopulations. The cofficient value for the cross-diffusion can be negative, zero or positive. Positive cross diffusion, $d_{s v}$, refers to movement of the susceptible, $S$ towards lower density of the infected $V$, while negative cross diffusion denotes susceptible, $s$ diffusing towards higher densities of the infected, $v$ [33]. The dynamics of interactive populations with self and cross-diffusion are investigated by researchers Levin [15] Levin and Segel, [16], Okubo and Levin [28], Mimura and Murray [26] and Mimura and Kawasaki [27].

In this paper, SIS mathematical model of malaria, including self and cross-diffusion in the system of differential equations, is studied. The main aim of this study is to consider transmission of disease under the impact of self and cross diffusion in the system. The SIS malaria model is solved numerically under various initial human and mosquito population distribution cases. Stability of the system, reproduction number and bifurcation values under disease free equilibrium(DFE) and endemic equilibrium(EE) are also considered.

\subsection{Formulation of the Model}

\section{The SIS Model in the Presence of Self and Cross-Diffusion}

This model is a generic compartment model on the mosquito-borne disease, malaria $[24,9,17,36,18,25]$. The human population is divided into the two compartments susceptible, $S$ and infectious, $I$. The density of the total human population is $N$, where $N=S+I$. The mosquito population is divided into the two compartments susceptible, $U$ and Infectious, $V$. The density of the total mosquito population is $M$, where $M=U+V$. The model consists of the following system of differential equations:

$$
\begin{aligned}
\frac{d S}{d t} & =b_{h} N-\alpha \frac{S V}{N}-\mu_{h} S+\delta I \\
\frac{d I}{d t} & =\alpha \frac{S V}{N}-\mu_{h} I-\delta I \\
\frac{d U}{d t} & =b_{m} M-\beta \frac{U I}{N}-\mu_{m} U \\
\frac{d V}{d t} & =\beta \frac{U I}{N}-\mu_{m} V
\end{aligned}
$$

where $S, I, U$ and $V$ express the densities of susceptible humans, infected humans, susceptible mosquitoes and infectious mosquitoes respectively. The biological meanings of the parameters and their values are given in Table 1.

Assume that $s, i, u$ and $v$, represent proportions of human and mosquito populations and $\psi$ reperesented as follows:

$s=\frac{S}{N}, i=\frac{I}{N}, u=\frac{U}{M}, v=\frac{V}{M}$ and $\psi=\alpha \frac{M}{N}$.

where $s+i=1$ and $u+v=1$. The system of differential equations with incorporating self and cross diffusion can be written as:

$$
\begin{aligned}
\frac{\partial s}{\partial t} & =b_{h}(1-s)-\psi s v+\delta i+d_{1} \frac{\partial^{2} s}{\partial x^{2}}+d_{s v} \frac{\partial^{2} v}{\partial x^{2}} \\
\frac{\partial i}{\partial t} & =\psi s v-\left(b_{h}+\delta\right) i+d_{2} \frac{\partial^{2} i}{\partial x^{2}}+d_{i u} \frac{\partial^{2} u}{\partial x^{2}} \\
\frac{\partial u}{\partial t} & =b_{m}(1-u)-\beta u i+d_{3} \frac{\partial^{2} u}{\partial x^{2}} \\
\frac{\partial v}{\partial t} & =\beta u i-b_{m} v+d_{4} \frac{\partial^{2} v}{\partial x^{2}}
\end{aligned}
$$

where $x$ is a spatial variable, $d_{1}$ and $d_{2}$ are the self-diffusion coefficients for humans, $d_{3}$ and $d_{4}$ are the diffusion coefficients for mosquitoes and, $d_{s v}, d_{i u}$ are the cross - diffusion coefficients.

Table 1: Interpretation of parameters

\begin{tabular}{|c|c|c|}
\hline Parameter & Interpretation & Values \\
\hline \hline$\alpha$ & Human infection rate & $0.4^{a}$ \\
\hline$\beta$ & Mosquito infection rate & $0.7^{a}$ \\
\hline$b_{h}$ & Human birth rate & $0.00005^{b}$ \\
\hline$b_{m}$ & Mosquito birth rate & $0.02^{b}$ \\
\hline$\mu_{h}$ & Human natural death rate & - \\
\hline$\mu_{m}$ & Mosquito natural death rate & - \\
\hline$\delta$ & Recovery rate of human & $0.01^{c}$ \\
\hline
\end{tabular}

${ }^{a}$ Vincent Margiotta, Lucas Oglesby, Teresa Portone and Brittany Stephenson[23]

${ }^{b}$ Nakul Chitnis, James M. Hyman and Jim M. Cushing[8].

${ }^{c}$ Lou, Yijun and Zhao, Xiao-Qiang[18]. 


\subsection{Boundary and initial conditions}

The domain of all the computations is considered as $[-2,2]$. The Boundary conditions are selected as follows:

$$
\begin{aligned}
& \frac{\partial s(-2, t)}{\partial x}=\frac{\partial i(-2, t)}{\partial x}=\frac{\partial u(-2, t)}{\partial x}=\frac{\partial v(-2, t)}{\partial x}=0 \\
& \frac{\partial s(-2, t)}{\partial x}=\frac{\partial i(-2, t)}{\partial x}=\frac{\partial u(-2, t)}{\partial x}=\frac{\partial v(-2, t)}{\partial x}=0
\end{aligned}
$$

The initial condition is as follows:

$$
\begin{array}{ll}
s_{0}=0.7 \exp \left(-(x+1)^{2}\right) & -2 \leqslant x \leqslant 2 \\
i_{0}=0.3 \exp \left(-(x+1)^{2}\right) & -2 \leqslant x \leqslant 2 \\
u_{0}=0.4 \exp \left(-5 x^{2}\right) & -2 \leqslant x \leqslant 2 \\
v_{0}=0.6 \exp \left(-5 x^{2}\right) & -2 \leqslant x \leqslant 2
\end{array}
$$

The graphs of the initial condition are shown in Fig 1. It shows the proportion of susceptible humans greater than the proportion of infected humans. The distribution of the human population is concentrated toward the left half of the main domain $[-2,2]$. The initial proportion of susceptible mosquitoes has been assumed to be less than the initial proportion of infected mosquitoes. The mosquito population is distributed symmetrically around the middle of the domain $[-2,2]$.

The values of the self-diffusion coefficients are chosen as, $d_{1}=0.05\left(\mathrm{~km}^{2} . d a y^{-1}\right), d_{2}=0.001\left(\mathrm{~km}^{2} . d a y^{-1}\right), d_{3}=0.0125$ $\left(\mathrm{km}^{2} . d a y^{-1}\right), d_{4}=0.0025\left(\mathrm{~km}^{2} . d a y^{-1}\right)$ and be all constant. The parameter values $\Delta x=0.1$ and $\Delta t=0.025$ are used in the numerical computations. Values of self and cross diffusion coefficients for the five different cases studied are given in Table 2.

Table 2: Diffusion Coefficient Values by Case $\left(\mathrm{km}^{2} . d a y^{-1}\right)$

\begin{tabular}{|ccccccc|}
\hline Case & $d_{1}$ & $d_{2}$ & $d_{3}$ & $d_{4}$ & $d_{s v}$ & $d_{i u}$ \\
\hline 1 & 0.0500 & 0.0010 & 0.0125 & 0.0025 & 0.0000 & 0.0000 \\
2 & 0.0500 & 0.0010 & 0.0125 & 0.0025 & +0.0150 & +0.012 \\
3 & 0.0500 & 0.0010 & 0.0125 & 0.0025 & -0.0150 & 0.0000 \\
4 & 0.0000 & 0.0000 & 0.0000 & 0.0000 & +0.0150 & +0.012 \\
5 & 0.0000 & 0.0000 & 0.0000 & 0.0000 & -0.0150 & 0.0000 \\
\hline
\end{tabular}

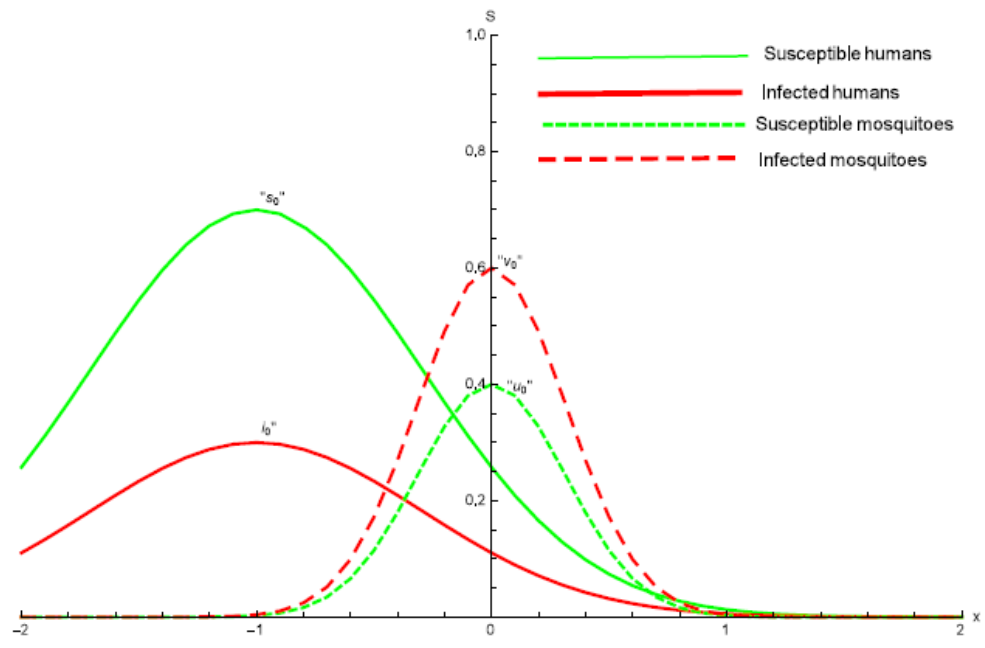

Figure 1: Initial condition

\subsection{Basic Reproduction Number without Diffusion}

The point of equilibrium for disease-free equilibrium is

$P_{0}(1,0,1,0)$

and the point of endemic equilibrium is

$$
\begin{aligned}
P^{*}\left(s^{*}, i^{*}, u^{*}, v^{*}\right) & =\left(\frac{\left(\delta+b_{h}\right)\left(\beta+b_{m}\right)}{\beta\left(\psi+\delta+b_{h}\right)}, \frac{\psi \beta-\delta b_{m}-b_{h} b_{m}}{\beta\left(\psi+\delta+b_{h}\right)}, \frac{\left(\psi+\delta+b_{h}\right) b_{m}}{\psi\left(\beta+b_{m}\right)}, \frac{\psi \beta-\delta b_{m}-b_{h} b_{m}}{\psi\left(\beta+b_{m}\right)}\right) \\
& =(0.025209,0.974791,0.028475,0.971524)
\end{aligned}
$$


One of the important aspects of epidemic models is the basic reproduction number, $R_{0}$. It indicates whether the disease will spread through the population or die out. It is given by the average number of secondary infectious cases infected by a "typical" infectious individual in a totally the susceptible population [20]. In order to calculate the basic reproduction number, the next generation matrix operator method described [35] is used. Here, matrix $\mathcal{F}$, the matrix of the new infection and $\nu$, the matrix of the transition between compartments are given as

$\mathcal{F}=\left(\begin{array}{c}\psi s v \\ \beta u i \\ 0 \\ 0\end{array}\right)$ and $\nu=\left(\begin{array}{c}\left(\delta+b_{h}\right) i \\ b_{m} v \\ 0 \\ 0\end{array}\right)$

The derivatives of $\mathcal{F}$ and $\nu$ at the point of disease - free equilibrium $P_{0}$ are $F$ and $V$ given by :

$F=\left(\begin{array}{cc}0 & \psi \\ \beta & 0\end{array}\right)$ and $V=\left(\begin{array}{cc}\delta+b_{h} & 0 \\ 0 & b_{m}\end{array}\right)$

The matrix $F V^{-1}$ is

$F V^{-1}=\left(\begin{array}{cc}0 & \frac{\psi}{b_{m}} \\ \frac{\beta}{\left(\delta+b_{h}\right)} & 0\end{array}\right)$

The basic reproduction number, $R_{0}$ is the dominant eigenvalue ( the spectral radius of the product) of $F V^{-1}$, so

$R_{0}=\sqrt{\frac{\psi \beta}{\left(\delta+b_{h}\right) b_{m}}}$

\subsection{Stability Analysis of Equilibria of SIS Model}

\subsubsection{Stability Analysis of Disease-Free Equilibrium (DFE) in the Absence of Diffusion}

In order to compute the stability of disease-free equilibrium, the variational matrix of the system equations (5) - (8), is calculated

$W=\left[\begin{array}{cccc}-b_{h}-\alpha \psi & \delta & 0 & -s \psi \\ \psi v & -\delta-b_{h} & 0 & s \psi \\ 0 & -u \beta & -i \beta-b_{m} & 0 \\ 0 & u \beta & i \beta & -b_{m}\end{array}\right]$

The variational matrix at the disease-free equilibrium point $P_{0}(1,0,1,0)$, is given

$$
W^{0}=\left[\begin{array}{cccc}
-b_{h} & \delta & 0 & -\psi \\
0 & -\delta-b_{h} & 0 & \psi \\
0 & -\beta & -b_{m} & 0 \\
0 & \beta & 0 & -b_{m}
\end{array}\right]
$$

The characteristic equation of this matrix $W^{0}$ at the point of free-equilibrium $P_{0}$, can be written as

$$
\lambda^{4}+p_{1} \lambda^{3}+p_{2} \lambda^{2}+p_{3} \lambda+p_{4}=0
$$

where

$p_{1}=\delta+2 b_{h}+2 b_{m}$,

$p_{2}=-\psi \beta+b_{h}^{2}+2 \delta b_{m}+b_{m}^{2}+b_{h}\left(\delta+4 b_{m}\right)$,

$p_{3}=2 b_{h} b_{m}+b_{m}\left(-\psi \beta+\delta b_{m}\right)+b_{h}\left(-\psi \beta+2 \delta b_{m}+2 b_{m}^{2}\right)$,

$p_{4}=b_{h} b_{m}\left(-\psi \beta+\left(\delta+b_{h}\right) b_{m}\right)$.

Here, $p_{1}, p_{2}, p_{3}$ and $p_{4}$ are computed using the technique applied by Chakraborty [7].

Routh-Hurwitz stability criteria are $p_{1}>0, p_{3}>0, p_{4}>0$ and

$$
\begin{aligned}
p_{1} p_{2} p_{3}-p_{3}^{2}-p_{1}^{2} p_{4}>0 & \Rightarrow-\psi \beta b_{h} b_{m}+\delta b_{h} b_{m}^{2}+b_{h}^{2} b_{m}^{2}>0 \\
& \Rightarrow-\psi \beta+\left(\delta+b_{h}\right) b_{m}>0 \\
& \Rightarrow \frac{\psi \beta}{\left(\delta+b_{h}\right) b_{m}}<1 .
\end{aligned}
$$

Thus the disease-free equilibrium is stable when $R_{0}=\sqrt{\frac{\psi \beta}{\left(\delta+b_{h}\right) b_{m}}}<1$.

Routh-Hurwitz stability conditions of disease-free equilibrium are given in Table 3.

Table 3: Routh-Hurwitz Stability criteria of Disease-Free Equilibrium

\begin{tabular}{ccccccc}
\hline Point of equilibrium & $p_{1}$ & $p_{2}$ & $p_{3}$ & $p_{4}$ & $p_{1} p_{2} p_{3}-p_{3}^{2}-p_{1}^{2} p_{4}$ & Stable/unstable \\
\hline$(1,0,1,0)$ & 0.0501 & 0.000804503 & $4.0601 \times 10^{-6}$ & $2.01 \times 10^{-10}$ & $1.46656 \times 10^{-10}$ & Stable
\end{tabular}

2.4.2 Stability Analysis of Endemic Equilibrium in the Presence of Diffusion

In order to study the stability of endemic equilibrium, the equations (5)-(8) are linearized about the point of equilibrium, $P^{*}$, to compute the small perturbations $s_{1}(x, t), i_{1}(x, t), u_{1}(x, t)$ and $v_{1}(x, t)$. 


$$
\begin{aligned}
\frac{\partial s_{1}}{\partial t} & =a_{11} s_{1}+a_{12} i_{1}+a_{13} u_{1}+a_{14} v_{1}+d_{1} \frac{\partial^{2} s_{1}}{\partial x^{2}}+d_{s v} \frac{\partial^{2} v}{\partial x^{2}} \\
\frac{\partial i_{1}}{\partial t} & =a_{21} s_{1}+a_{22} i_{1}+a_{23} u_{1}+a_{24} v_{1}+d_{1} \frac{\partial^{2} s_{1}}{\partial x^{2}}+d_{i u} \frac{\partial^{2} u}{\partial x^{2}} \\
\frac{\partial u_{1}}{\partial t} & =a_{31} s_{1}+a_{32} i_{1}+a_{33} u_{1}+a_{34} v_{1}+d_{3} \frac{\partial^{2} u_{1}}{\partial x^{2}} \\
\frac{\partial v_{1}}{\partial t} & =a_{41} s_{1}+a_{42} i_{1}+a_{43} u_{1}+a_{44} v_{1}+d_{4} \frac{\partial^{2} v_{1}}{\partial x^{2}}
\end{aligned}
$$

where $a_{11}, a_{12}, a_{13}$ etc. are the elements of the variational matrix $\mathrm{V}^{*}$ computed by using a method similar that of [31]. Suppose a Fourier series solution exists of Eqs.(12)-(15) of the form

$$
\begin{aligned}
& s_{1}(x, t)=\sum s_{k} e^{\lambda t} \cos (k x), \\
& i_{1}(x, t)=\sum i_{k} e^{\lambda t} \cos (k x), \\
& u_{1}(x, t)=\sum u_{k} e^{\lambda t} \cos (k x), \\
& v_{1}(x, t)=\sum v_{k} e^{\lambda t} \cos (k x),
\end{aligned}
$$

where $k=\frac{n \pi}{2},(n=1,2,3, \ldots)$ is the wave number for node $n$. Substituting the values of $s_{1}, i_{1}, u_{1}$ and $v_{1}$ in the Eqs. (12)-(15), the equations are transformed into

$$
\begin{aligned}
& \sum_{k}\left(a_{11}-d_{1} k^{2}-\lambda\right) s_{k}+\sum_{k} a_{12} i_{k}+\sum_{k} a_{13} u_{k}+ \\
& \sum_{k}\left(a_{14}-d_{s v} k^{2}\right) v_{k}=0, \\
& \sum_{k} a_{21} s_{k}+\sum_{k}\left(a_{22}-d_{2} k^{2}-\lambda\right) i_{k}+\sum_{k}\left(a_{23}-d_{i u} k^{2}\right) u_{k}+ \\
& \sum_{k} a_{24} v_{k}=0, \\
& \sum_{k} a_{32} i_{k}+\sum_{k}\left(a_{33}-d_{3} k^{2}-\lambda\right) u_{k}=0, \\
& \sum_{k} a_{42} i_{k}+\sum_{k} a_{43} u_{k}+\sum_{k}\left(a_{44}-d_{4} k^{2}-\lambda\right) v_{k}=0,
\end{aligned}
$$

The variational matrix $V^{d}$ for the Eqs. (20)-(23) is

$$
V^{d}=\left[\begin{array}{cccc}
a_{11}-d_{1} k^{2} & a_{12} & a_{13} & \left(a_{14}-d_{s v} k^{2}\right) \\
a_{21} & a_{22}-d_{2} k^{2} & a_{23}-d_{i u} k^{2} & a_{24} \\
0 & a_{32} & a_{33}-d_{3} k^{2} & 0 \\
0 & a_{42} & a_{43} & a_{44}-d_{4} k^{2}
\end{array}\right]
$$

The characteristic equation for the variational matrix $V^{d}$ can be written as

$$
\lambda^{4}+g_{1} \lambda^{3}+g_{2} \lambda^{2}+g_{3} \lambda+g_{4}=0
$$

where $g_{1}, g_{2}, g_{3}$ and $g_{4}$ are computed with the technique used in [7]. The Routh-Hurwitz conditions are given as: $g_{1}>0, g_{3}>0, g_{4}>0$ and $g_{1} g_{2} g_{3}-g_{3}^{2}-g_{1}^{2} g_{4}>0$.

Numerical calculations of the Routh-Hurwitz conditions for Cases 1,2 and 3 at the point of equilibrium considered are given in Table 4

$P^{*}=(0.025209,0.974791,0.028475,0.971524)$

Table 4: Routh-Hurwitz Stability criteria of equilibria

\begin{tabular}{ccccccc}
\hline Case & $g_{1}$ & $g_{2}$ & $g_{3}$ & $g_{4}$ & $g_{1} g_{2} g_{3}-g_{1}^{2} g_{4}-g_{3}^{2}$ & Stable/unstable \\
\hline 1 & 1.28391 & 0.419831 & 0.0119453 & 0.0000471502 & 0.00621841 & Stable \\
2 & 1.28391 & 0.419733 & 0.0120357 & 0.0000531203 & 0.00654333 & Stable \\
3 & 1.28391 & 0.419621 & 0.0118019 & 0.0000398621 & 0.00643513 & Stable
\end{tabular}

The basic reproduction number with self and cross-diffusion, $R_{0}^{d}$ is obtained the same way as described in subsection 2.3 , where

$R_{0}^{d}=\sqrt{\frac{\beta\left(d_{3} k^{2} \alpha+\psi b_{m}\right)}{\left(d_{4} k^{2} \psi+b_{m}\right)\left(d_{2} d_{3} k^{4}-d_{i u} * k^{2} \beta+d_{3} k^{2} \delta+d_{3} k^{2} b_{h}+d_{2} k^{2} b_{m}+\delta b_{m}+b_{h} b_{m}\right)}}$

Values of the reproductive numbers for Cases 1,2 and 3 considered in the Table 2 are given in Table 5. 
Table 5: Basic Reproductive Number, $R_{0}^{d}$

\begin{tabular}{|c|c|}
\hline Case & values of $R_{0}^{d}$ \\
\hline 1 & 9.95542 \\
\hline 2 & 10.7489 \\
\hline 3 & 10.6399 \\
\hline
\end{tabular}

\subsection{Excited Mode and Bifurcation Value}

In order to determine the first excited mode of oscillations $n$, the same technique is applied as used in $[7 \mid$. In Case $1, n=1$ is the first mode of excitationit being closest to the vertical axis with the least value of $\alpha$, of the function $f(k)=g_{1} g_{2} g_{3}-g_{3}^{2}-g_{1}^{2} g_{4}$ Similarly, $n=1$ is the first mode of excitation for Cases 2 and 3 .

Bifurcation values of the human transmission rate, $\alpha$ and bifurcation values of human recovery rate, $\delta$ are given in Table 6 .

Table 6: Bifurcation Values of the Parameters $\alpha$ and $\delta$ with Self and Cross-

\begin{tabular}{|c|c|c|c|c|}
\hline \multirow{2}{*}{ Cases } & \multicolumn{2}{|c|}{ Original values } & \multicolumn{2}{c|}{ Bifurcation values } \\
\cline { 2 - 5 } & $\alpha$ & $\delta$ & $\alpha$ & $\delta$ \\
\hline 1 & 0.4000 & 0.01 & 0.44713 & 0.01017 \\
2 & 0.4000 & 0.01 & 0.37620 & 0.01321 \\
3 & 0.4000 & 0.01 & 0.35231 & 0.01010 \\
\hline
\end{tabular}

Diffusion

- In Case1, with self diffusion the bifurcation value of human infection rate, $\alpha$ is greater than the corresponding with self and cross-diffusion as in Case 2 and 3.

- In Case 2, where cross-diffusion $d_{s v}$ and $d_{i u}$ have positive values, the bifurcation value of the transmission rate of human infection with cross diffusion is smaller than the corresponding value in Case 1.

- There is a sharp increase in the bifurcation values of human infection rate, $\alpha$ in Case 1 . This implies that the system remains stable for higher value of human infection rate, $\alpha$ with Case 1.

- The human recovery rate, $\delta$, stabilises the system at lower bifurcation values in Cases 1 and 3 than in Case 2 .

\subsection{Numerical scheme}

The operator splitting technique $[12,32,37]$ has been applied to solve the SIS malaria model equations. The equations are divided into two parts as sub-equations. The first part is the set of nonlinear reaction equations, which are considered for the first half of the time step as follows:

$$
\begin{aligned}
\frac{1}{2} \frac{\partial s}{\partial t} & =b_{h}(1-s)-\psi s v+\delta i, \\
\frac{1}{2} \frac{\partial i}{\partial t} & =\psi s v-\left(b_{h}+\delta\right) i, \\
\frac{1}{2} \frac{\partial u}{\partial t} & =b_{m}(1-u)-\beta u i, \\
\frac{1}{2} \frac{\partial v}{\partial t} & =\beta u i-b_{m} v,
\end{aligned}
$$

The second part of the system of equations consists of the linear diffusion equations, to be used for the second half-time step:

$$
\begin{aligned}
\frac{1}{2} \frac{\partial s}{\partial t} & =d_{1} \frac{\partial^{2} s}{\partial x^{2}}+d_{s v} \frac{\partial^{2} v}{\partial x^{2}} \\
\frac{1}{2} \frac{\partial i}{\partial t} & =d_{2} \frac{\partial^{2} i}{\partial x^{2}}+d_{i u} \frac{\partial^{2} u}{\partial x^{2}} \\
\frac{1}{2} \frac{\partial u}{\partial t} & =d_{3} \frac{\partial^{2} u}{\partial x^{2}} \\
\frac{1}{2} \frac{\partial v}{\partial t} & =d_{4} \frac{\partial v^{2}}{\partial x^{2}} .
\end{aligned}
$$

The Forward Euler scheme is used with the above system of equations to convert to

$$
\begin{aligned}
s_{k}^{j+\frac{1}{2}} & =s_{k}^{j}+\Delta t\left(b_{h}\left(1-s_{k}^{j}\right)-\psi s_{k}^{j} v_{k}^{j}+\delta i_{k}^{j}\right), \\
i_{k}^{j+\frac{1}{2}} & =i_{k}^{j}+\Delta t\left(\psi s_{k}^{j} v_{k}^{j}-\left(b_{h}-\delta\right) i_{k}^{j}\right), \\
u_{k}^{j+\frac{1}{2}} & =u_{k}^{j}+\Delta t\left(b_{m}\left(1-u_{k}^{j}\right)-\beta u_{k}^{j} i_{k}^{j}\right), \\
v_{k}^{j+\frac{1}{2}} & =v_{k}^{j}+\Delta t\left(\beta u_{k}^{j} i_{k}^{j}-b_{m} v_{k}^{j}\right),
\end{aligned}
$$


where $s_{k}^{j}, i_{k}^{j}, u_{k}^{j}$ and $v_{k}^{j}$ are the approximated values of $s, i, u$ and $v$ at position $-2+k \Delta x$ for $k=0,1, \ldots$ and time $j \Delta t$, $j=0,1, \ldots$ and $s_{k}^{j+\frac{1}{2}}, i_{k}^{j+\frac{1}{2}}, u_{k}^{j+\frac{1}{2}}$ and $v_{k}^{j+\frac{1}{2}}$ represent the values at the half-time step. In the same way, for the second half-time step,

$$
\begin{aligned}
s_{k}^{j+\frac{1}{2}}= & s_{k}^{j+\frac{1}{2}}+d_{1} \frac{\triangle t}{(\triangle x)^{2}}\left(s_{k-1}^{j+\frac{1}{2}}-2 s_{k}^{j+\frac{1}{2}}+s_{k+1}^{j+\frac{1}{2}}\right)+ \\
& d_{s v}\left(v_{k-1}^{j+\frac{1}{2}}-2 v_{k}^{j+\frac{1}{2}}+v_{k+1}^{j+\frac{1}{2}}\right), \\
i_{k}^{j+\frac{1}{2}}= & i_{k}^{j+\frac{1}{2}}+d_{2} \frac{\triangle t}{(\triangle x)^{2}}\left(i_{k-1}^{j+\frac{1}{2}}-2 i_{k}^{j+\frac{1}{2}}+i_{k+1}^{j+\frac{1}{2}}\right)+ \\
& d_{i u}\left(u_{k-1}^{j+\frac{1}{2}}-2 u_{k}^{j+\frac{1}{2}}+u_{k+1}^{j+\frac{1}{2}}\right), \\
u_{k}^{j+\frac{1}{2}}= & u_{k}^{j+\frac{1}{2}}+d_{3} \frac{\triangle t}{(\triangle x)^{2}}\left(u_{k-1}^{j+\frac{1}{2}}-2 u_{k}^{j+\frac{1}{2}}+u_{k+1}^{j+\frac{1}{2}}\right), \\
v_{k}^{j+\frac{1}{2}}= & v_{k}^{j+\frac{1}{2}}+d_{4} \frac{\triangle t}{(\triangle x)^{2}}\left(v_{k-1}^{j+\frac{1}{2}}-2 v_{k}^{j+\frac{1}{2}}+v_{k+1}^{j+\frac{1}{2}}\right),
\end{aligned}
$$

The stability condition for numerical solution of the differential equations is

$$
\frac{d_{n} \Delta t}{(\Delta x)^{2}} \leqslant 0.5, n=1,2,3,4,5 .
$$

The values $d_{1}=0.05\left(\mathrm{~km}^{2} . d a y^{-1}\right), d_{2}=0.001\left(\mathrm{~km}^{2} . d a y^{-1}\right), d_{3}=0.0125\left(\mathrm{~km}^{2} . d a y^{-1}\right)$,

$d_{4}=0.0025\left(\mathrm{~km}^{2} . d a y^{-1}\right), d_{s v}=0.0150\left(\mathrm{~km}^{2} . d a y^{-1}\right), d_{i u}=0.012\left(\mathrm{~km}^{2} . d a y^{-1}\right), \Delta x=0.1$ and $\triangle t=0.025$ are used in each case considered here.

\subsection{Numerical Solutions}

In this section the numerical solutions of the differential equations including self and cross-diffusion in cases with three different cases of values for cross-diffusion, $d_{s v}$ and $d_{i u}$ are considered. Figs.2-4 illustrate the following results.

\subsubsection{Case 1}

Fig. 2, illustrates the outcome ofthe numerical solution with self-diffusion only. In this case, it is observed that the population of humans and mosquitoes spread over the domain $[-2,2]$. There is a considerable decrease during the first five days in the population of susceptible humans in the domain $[-1.6,0.4]$. After that, the decrease in this proportion continues until $t=20$ days in the domain $[-2,1]$. In the domain $[1,2]$, the proportion of susceptible humans increases slightly during the first five days and after that this proportion stays at the same level, as shown in Fig. 2(a). The human infected population increases befor $t=5$ days in the domain $[-1,1]$, but outside this domain there is not significant growth. After that, in the domain $[-2,0]$, the population spreads steadily. In the domain [0,1], the increase in proportions gets slower after $t=10$ days, as shown in Fig. $2(b)$. The proportion of susceptible mosquitoes declines dramatically in the first five days, in the domain [-0.5,0.4] as shown in Fig. $2(c)$. After $t=10$ days, a slow decrease occurs in the domain $[-0.6,0.4]$. In the domain $[-2,-0.6]$, the proportion of susceptibles starts to increase during the ten five days, after that this proportion slightly reduces in this period. In the domain [0.6,2], there is a significant increase with the passage of time as shown in Fig. 2(c). Meanwhile, the infected proportion of mosquitoes spreads toward the left side of the domain $[-2,2]$ as shown in Fig. 2(d). The infected proportion of mosquitoes increases significantly until $t=5$ days. This increase becomes very slow after $t=10$ days in the domain $[-0.4,0.2]$, as indicated by the peak values. Outside this domain there is some increase during the twenty days, as shown in Fig. $2(d)$.

\subsubsection{Case 2}

Fig. 3, illustrates the outcome of the numerical solution with self and cross-diffusion. Here cross diffusion $d_{s v}$ and $d_{i u}$ have positive values, meaning that both of susceptible and infected human populations move to lower densities of susceptible and

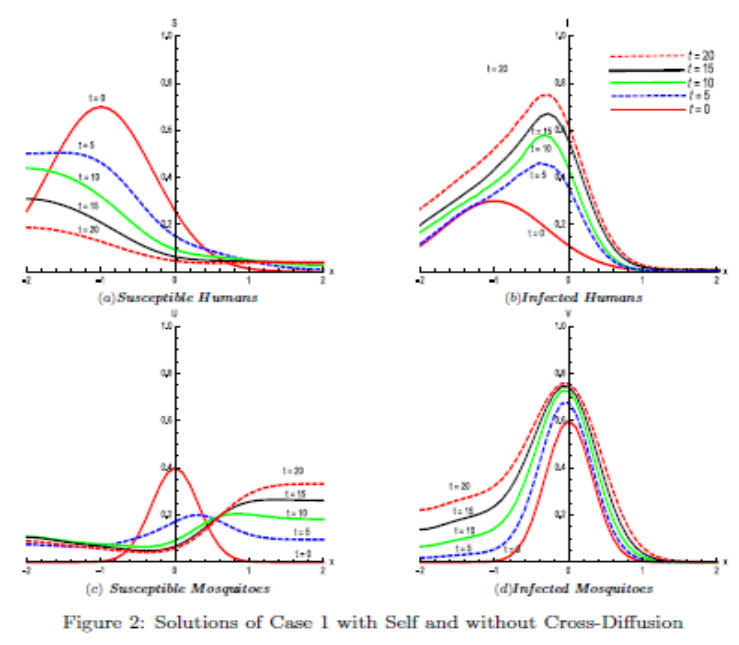


infected mosquito populations, respectively. There is an increased proportion of susceptible human as compared to Case 1 without cross-diffusion. An upward trend is recorded in the growth of infected humans at the peak. There is also significant change in the pattern formation with fluctuation over the domain $[0,1.2]$ compared to Case 1 . There is also a decrease in the infected proportion of mosquitoes as compared to Case 1, as shown in Fig.3(d).

\subsubsection{Case 3}

Fig. 4, illustrates the outcome of the numerical solution with self and cross-diffusion $d_{s v}$. The value of $d_{s v}$ is negative here, causing the a movement of the susceptible human population towards higher concentrations of infected mosquitoes. The proportion of susceptible humans declines significantly over the domain $[-2,0.2]$. The proportion of infected humans, under the effect of negative cross diffusion, has increased remarkably over the period 20 day period as shown in Fig. 4(b). .As shown in Fig. 4(d), the proportion of infected mosquitoes increases considerably in the first ten days, and after that there is not much increase in the domain $[-0.5,0.2]$.

\section{The SIS Model in the Presence of Cross-Diffusion Only}

In this section, the effect of cross-diffusion without self-diffusion on the spread of disease is considered for two different cases, Case 4 and Case 5. The values $\Delta x=0.1$ and $\Delta t=0.025$ are usedfor the parameters of the numerical computation. The values of the cross-diffusion coefficients $d_{s v}$, and $d_{i u}$ are taken as given in Table2.

\subsection{Stability Analysis of Equilibria of SIS Malaria Model with Cross-Diffusion Only.}

In order to analyse the stability of endemic equilibrium in the presence of cross diffusion, the Routh Hurwitz criteria are given as:

$c_{1}>0, c_{3}>0, c_{4}>0$ and $c_{1} c_{2} c_{3}-c_{3}^{2}-c_{1}^{2} c_{4}>0:$

Numerical values of the Routh Hurwitz criteria for each of cases at the point of equilibrium $P_{1}=(0.025209,0.974791,0.028475$, $)$ are given in Table 7.

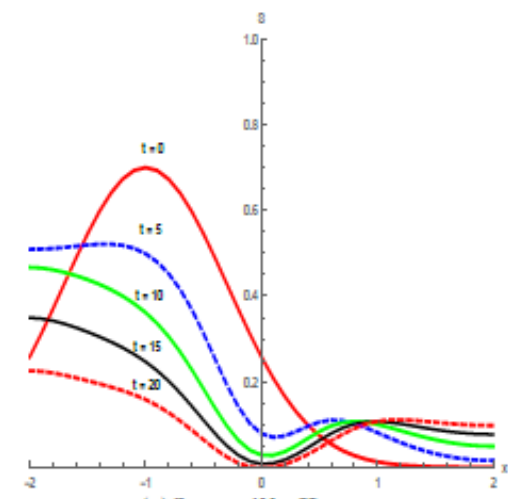

(a) Susceptible Humans

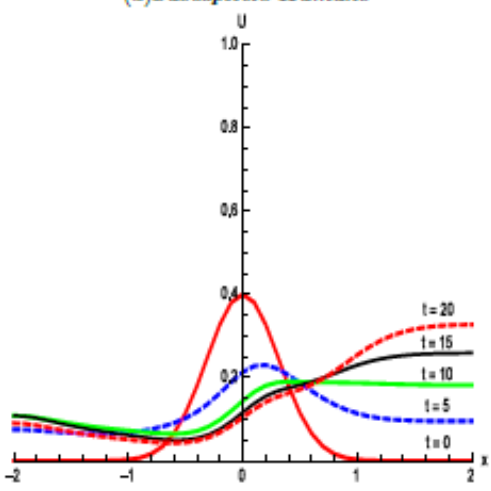

(c) Susceptible Mosquitoes

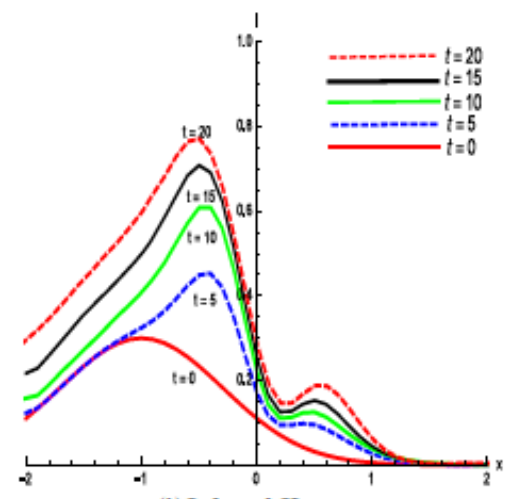

(b)Infected Humans

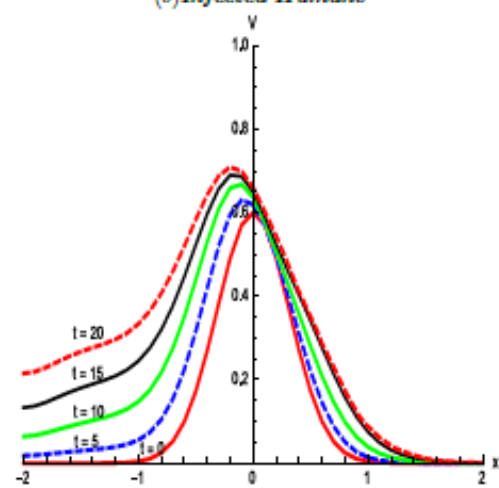

(d)Infected Mosquitoes

Figure 3: Solutions of Case 2 with Self and Cross-Diffusion 


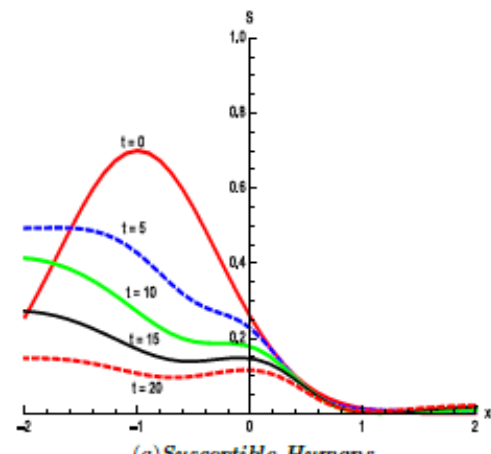

(a) Susceptible Human.

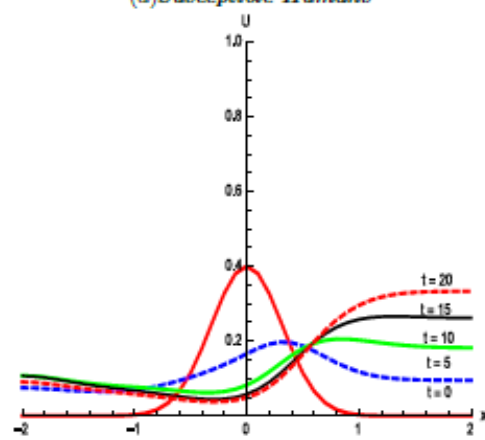

(c) Susceptible Mosquitoes
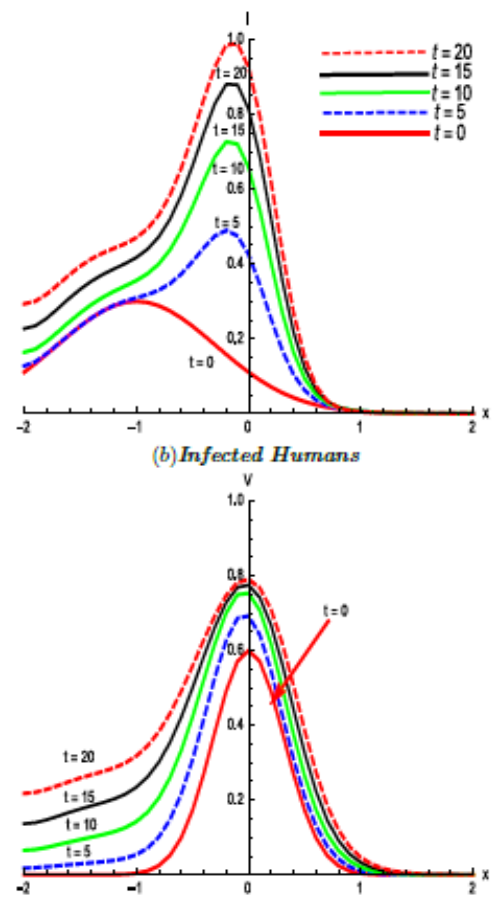

(d)Infected Mosquitoes

Figure 4: Solutions of Case 3 with Self and Cross-Diffusion

Table 7: Routh-Hurwitz stability criteria of equilibria with cross-diffusion only.

\begin{tabular}{ccccccc} 
Case & $c_{1}$ & $c_{2}$ & $c_{3}$ & $c_{4}$ & $c_{1} c_{2} c_{3}-c_{1}^{2} c_{4}-c_{3}^{2}$ & Stable/unstable \\
\hline 4 & 1.12106 & 0.301777 & 0.00571422 & $2.38212 \times 10^{-6}$ & 0.00189754 & Stable \\
5 & 1.12106 & 0.301875 & 0.00546772 & $-2.35713 \times 10^{-6}$ & 0.00182375 & Unstable
\end{tabular}

\subsection{Basic Reproduction Number with Cross-Diffusion}

The reproduction number with cross diffusion, $R_{0}^{c_{d}}$, is obtained using the same technique as given in Section 2 . Thus the reproduction number, with cross-diffusion only is:

$$
R_{0}^{c_{d}}=\sqrt{\frac{\psi b_{m}}{b_{m}\left(d_{i u} * k^{2} \beta+\delta b_{m}+b_{h} b_{m}\right)}}
$$

The values of $R_{0}^{c_{d}}$ for Cases 4 and 5 are given in Table 8.

Table 8: Basic Reproductive Number, $R_{0}^{c_{d}}$

\begin{tabular}{|c|c|}
\hline Case & Value of $R_{0}^{c_{d}}$ \\
\hline 4 & 10.0092 \\
\hline 5 & 10.3489 \\
\hline
\end{tabular}

\subsection{Bifurcation value}

Bifurcation values of the human transmission rate, $\alpha$ and human recovery rate, $\delta$ are given in Table 9 .

In Case 4 the bifurcation value of human infection rate, $\alpha$, is smaller than the bifurcation value of human infection in Case 5. This implies that the system remains stable for lower value of human infection rate, $\alpha$ with Case 4 , while the system becomes stable with higher bifurcation value in Case 5 .

Also, the bifurcation value of human recovery rate $\delta$, in Case 4 is greater than the bifurcation value of human recovery rate in Case 5. That means the system stabilises with higher bifurcation values of human recovery rate in Case 4 than in Case 5.

Table 9: Bifurcation Values of $\alpha$ and $\delta$ with Cross Diffusion

\begin{tabular}{|c|c|c|c|c|}
\hline \multirow{2}{*}{ Cases } & \multicolumn{2}{|c|}{ value chosen } & \multicolumn{3}{|c|}{ Bifurcation value } \\
\cline { 2 - 5 } & $\alpha$ & $\delta$ & $\alpha$ & $\delta$ \\
\hline 4 & 0.4000 & 0.01 & 0.40104 & 0.01211 \\
5 & 0.4000 & 0.01 & 0.57120 & 0.01001 \\
\hline
\end{tabular}




\subsection{Numerical Solutions}

Numerical solutions of the differential equations (5)-(8) with the inclusion in the system of cross-diffusion only have been obtained for two cases with different values $d_{s v}$ and $d_{i u}$ pair.

\subsubsection{Case 4}

Fig. 5, illustrates the outcome of the numerical solution for Case 4 with cross diffusion only. Here cross-diffusion coefficients, $d_{s v}$ and $d_{i u}$ have positive, meaning that both susceptible and infected human proportions move to areas lower of density of susceptible and infected mosquitoes, respectively. The proportions of susceptible human proportions are shown in Fig. $5(a)$. Over the domain $[-2,0.4]$, there is a decrease in proportion of susceptible human during the first five days. This decrease in proportions continues until $t=20$ days. During the same time, the proportion of susceptible humans increases slightly in the domain $[1.2,2]$ as shown in Fig. 5(a). However, the proportion of susceptible mosquitoes over the domain [-0.5,0.5] decreases significantly during the first five days as shown in Fig. $5(\mathrm{ca})$. There is also some reduction in the proportion of susceptible mosquitoes between $t=5$ and $t=10$ days. Regarding the infected mosquitoes, the proportion increases with the passage of time, but the most significant increase occurs during the first ten days, as shown in Fig.5(d).
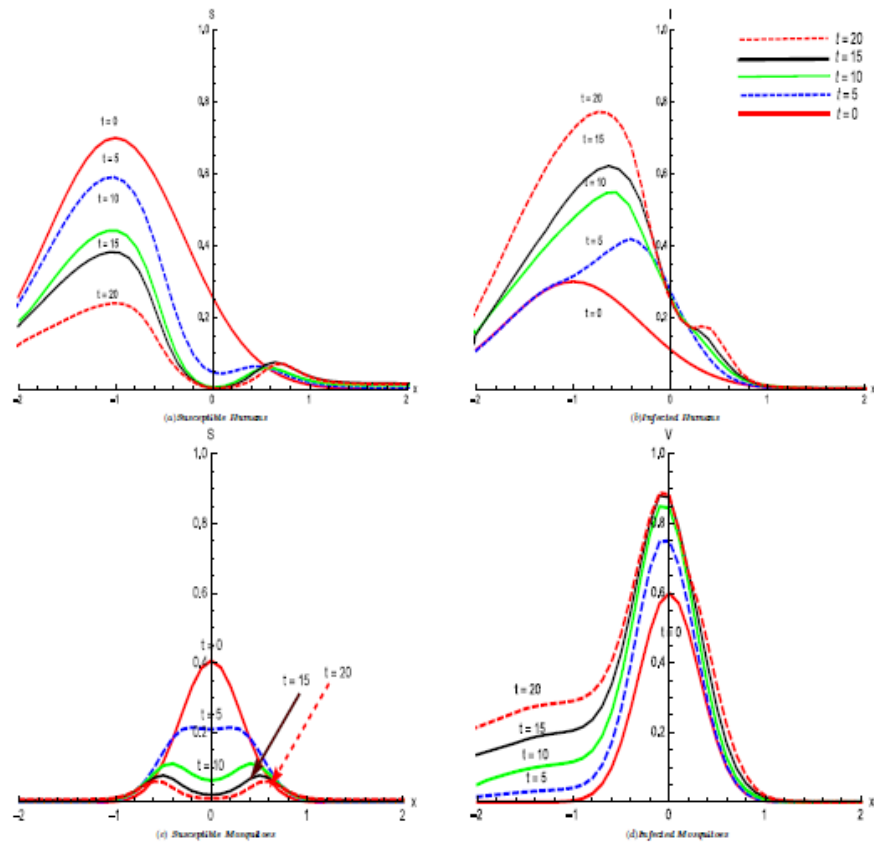

Figure 5: Solutions of Case 4 with Cross-Diffusion Only
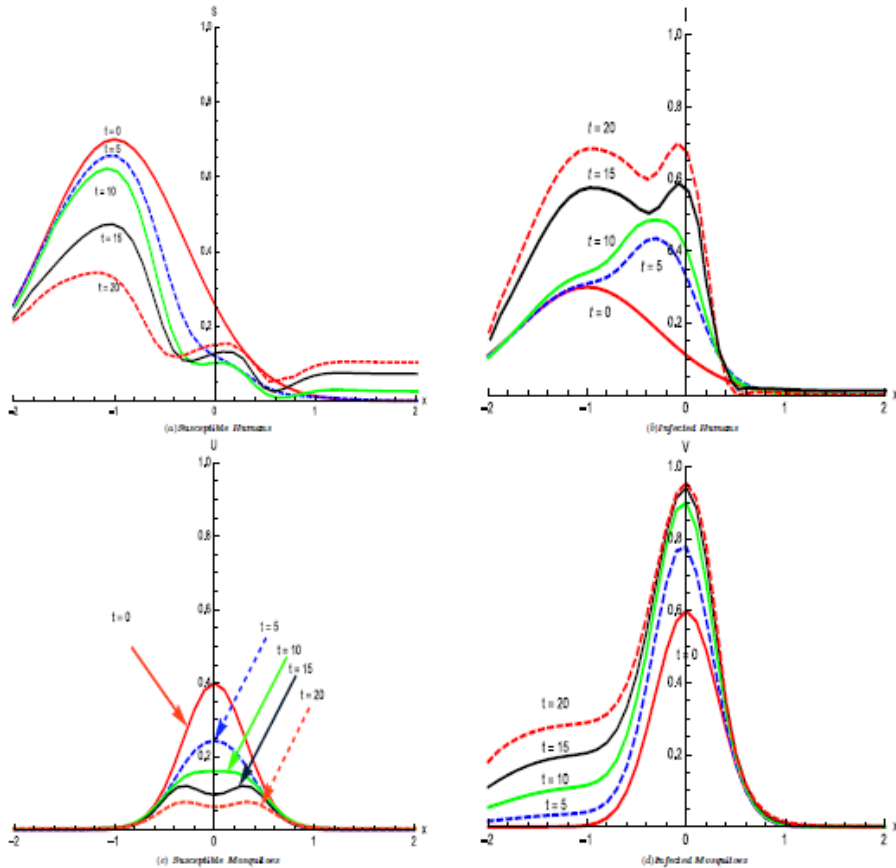

Figure 6: Solutions of Case 5 with Cross-Diffusion Only 


\subsubsection{Case 5}

Fig. 6 shows the outcome of the numerical solution for Case 5 with cross diffusion only. Here cross diffusion $d_{s v}$ is negative. This implies that the susceptible human population moves to a domain of higher infected mosquitoes density. The effect of negative cross-diffusion in the system on the proportion of infected humans is evident in Fig. 6(b). The overall trend shows fluctuation with a remarkable change in the structure of the proportion of infected humans. In the process, susceptible mosquitoes also get infected becuase of the increase of infected human proportion in the domain $[-1,1]$. Thus there is increase in the proportions of infected mosquitoes as compared to Case 4 .

\section{Discussion and Conclusion}

An SIS malaria model with the inclusion of self and cross-diffusion in the system has been studied numerically. Three different cases with the inclusion of self and cross-diffusion coefficients in the system are considered. Two cases of pure cross-diffusion in the system are examined under the same initial population distribution. The results have been compared to observe the effects of self and cross-diffusion on the population distribution of infected humans and mosquitoes leading to the spread of malaria disease. Reproduction numbers are obtained both for disease-free(DFE) and endemic equilibrium(EE). The stability of the equilibrium with DFE and EE is established using the Routh-Hurwitz stability criterion. The bifurcation values of human infection rate, $\alpha$ and recovery rate, $\delta$ are also calculated.

In presence of only self-diffusion in the system as in Case 1, a considerable reduction in the proportion of susceptible human and mosquito populations is observed. There is significant change in the pattern of distribution of their proportions along with spread all over the domain. Infected proportions of both humans and mosquitoes have increased with a spread all over the domain $[-2,2]$.

In Case 2, where both self and cross-diffusion are present in the system with $d_{s v}$ and $d_{i u}$ having positive values, the susceptible and infected human populations move to a lower-densities of infected and susceptible mosquitoes, respectively. During the period of 20 days, the susceptible proportion of humans decreases considerably in the domain $[-2,0]$ as compared to Case 1 , without cross-diffusion. The susceptible mosquito proportion decreases and the pattern of distribution is fairly close to Case 1 . The proportion of infected mosquitoes is observed moving to the left of the domain $[-2,2]$ with a reduced proportion of the spread under the impact of the cross diffusion for the susceptible human to the infected mosquitoes during the same time as compared to Case 1.

In Case 3, with self-diffusion and negative cross diffusion $d_{s v}$, the proportions infected of humans and mosquitoes surge considerably during the time period of 20 days as compared to other cases.

In Case $4, d_{s v}$ and $d_{i u}$, have positive values. There is a significant decrease in the proportion of susceptible humans and mosquitoes until $t=10$ days, thereafter remaining steady in the domain $[-2,0]$. The proportions of the infected population of humans and mosquito have a substantial increase in the domain $[-2,0]$.

In Case 5 , the cross diffusion, $d_{s v}$ is negative and $d_{i u}$ is equal zero. The proportion of susceptible humans has markedly decreased, with the some fluctuations. The proportion of infected humans exhibts considerable influence of negative cross diffusion on the infected population. The infected humans has increased and fluctuates in the domain [-1,0.4]. The mosquito proportion shows the same behaviour as with and without self-diffusion in the system as in Cases 1,2 and 3 .

Numerical values of reproduction number for all cases have been calculated. Reproduction number is minimised in the absence of cross diffusion, thus showing that the disease spreads slowly as compared to other cases where both cross diffusion along with self diffusion are present in the system. The numerical results show that the self-diffusion and cross-diffusion of the population have great impact on the dynamics of population density variation and thus influence the spread of the malaria epidemic.

The results can be summarised as

- The system remains stable with considerable higher values of infection rate, $\alpha$, with self-diffusion. Thus self-diffusion is responsible for accelerating the spread of disease.

- The system remains stable with the reduced values of infection rate, $\alpha$ under self and negative cross-diffusion. Thus self and cross-diffusion together in the system decelerate the spread of disease.

- The system remains stable with the highest value of $\alpha$, with pure negative cross-diffusion in the system, thus promoting the highest rate of spread of the disease.

- The system remains stable with the highest value of recovery rate, $\delta$ with self and positive cross-diffusion. Thus maximum recovery is possible with self and positive cross-diffusion in the system.

- The system remains stable with the lowest value of recovery rate, $\delta$ with pure negative cross- diffusion. Thus minimum recovery is possible with pure negative cross-diffusion in the system.

- Negative cross- diffusion in the system introduced the instability in the system.

- Initial distribution of populations of humans and mosquitoes does make a difference to the spread of the disease.

- Reproduction numbers show that malaria disease has considerable chances of becoming endemic with the inclusion of cross-diffusion along with self diffusion in the system. 


\section{$5 \quad$ Acknowledgments}

One of the authors, Bushra Hasan is thankful to Swinburne University of Technology, Melbourne, Australia for providing the necessary facilities to carry on this work. Also thankful to to Republic of Iraq, Ministry of Higher Education and Scientific Research for the postgraduate research award.

\section{References}

[1] Centers for diseease control and prevention. cdc - malaria (2012).

[2] World malaria report 2014, fact sheet.

[3] Alfred Amambua-Ngwa, Kevin KA Tetteh, Magnus Manske, Natalia Gomez-Escobar, Lindsay B Stewart, M Elizabeth Deerhake, Ian H Cheeseman, Christopher I Newbold, Anthony A Holder, Ellen Knuepfer, et al., Population genomic scan for candidate signatures of balancing selection to guide antigen characterization in malaria parasites, PLoS Genet 8 (2012), no. $11, \mathrm{e} 1002992$.

[4] Joan L Aron, Mathematical modelling of immunity to malaria, Mathematical Biosciences 90 (1988), no. 1, 385-396.

[5] Norman TJ Bailey et al., The mathematical theory of infectious diseases and its applications, Charles Griffin \& Company Ltd, 5a Crendon Street, High Wycombe, Bucks HP13 6LE., 1975.

[6] Albert O Bush, Parasitism: the diversity and ecology of animal parasites, Cambridge University Press, 2001.

[7] Aspriha Chakraborty, Manmohan Singh, David Lucy, and Peter Ridland, Predator-prey model with prey-taxis and diffusion, Mathematical and computer modelling 46 (2007), no. 3, 482-498.

[8] Nakul Chitnis, James M Hyman, and Jim M Cushing, Determining important parameters in the spread of malaria through the sensitivity analysis of a mathematical model, Bulletin of mathematical biology 70 (2008), no. 5, 1272-1296.

[9] C Cosner, JC Beier, RS Cantrell, D Impoinvil, L Kapitanski, MD Potts, A Troyo, and S Ruan, The effects of human movement on the persistence of vector-borne diseases, Journal of theoretical biology 258 (2009), no. 4, 550-560.

[10] K Dietz, L Molineaux, and A Thomas, A malaria model tested in the african savannah, Bulletin of the World Health Organization 50 (1974), no. 3-4, 347.

[11] Denise L Doolan, Carlota Dobano, and J Kevin Baird, Acquired immunity to malaria, Clinical microbiology reviews 22 (2009), no. 1, 13-36.

[12] ALAN Easton, MANMOHAN Singh, and GURONG Cui, Solutions of two species reaction-diffusion systems, Can. Appl. Math. Quart 5 (1997), 359-373.

[13] Kate Grimwade, Neil French, Daniel D Mbatha, Dawn D Zungu, Martin Dedicoat, and Charles F Gilks, Hiv infection as a cofactor for severe falciparum malaria in adults living in a region of unstable malaria transmission in south africa, Aids 18 (2004), no. 3, 547-554.

[14] Yunfeng Jia and Pan Xue, Effects of the self-and cross-diffusion on positive steady states for a generalized predator-prey system, Nonlinear Analysis: Real World Applications 32 (2016), 229-241.

[15] Simon A Levin, A more functional response to predator-prey stability, American Naturalist (1977), 381-383.

[16] Simon A Levin and Lee A Segel, Hypothesis for origin of planktonic patchiness, (1976).

[17] Yijun Lou and Xiao-Qiang Zhao, The periodic ross-macdonald model with diffusion and advection, Applicable Analysis 89 (2010), no. 7, 1067-1089.

[18] _ A reaction-diffusion malaria model with incubation period in the vector population, Journal of mathematical biology $62(2011)$, no. $4,543-568$.

[19] Yuan Lou and Wei-Ming Ni, Diffusion vs cross-diffusion: an elliptic approach, Journal of Differential Equations 154 (1999), no. 1, 157-190.

[20] Zhien Ma, Yicang Zhou, and Jianhong Wu, Modeling and dynamics of infectious diseases, Higher Education Press, 2009.

[21] George Macdonald, The analysis of equilibrium in malaria., Tropical diseases bulletin 49 (1952), no. 9, 813-829.

[22] George Macdonald et al., The epidemiology and control of malaria., The Epidemiology and Control of Malaria. (1957).

[23] V. Margiotta et al., Analysis of the spread of malaria disease, Science (2010).

[24] Maia Martcheva and Olivia Prosper, Unstable dynamics of vector-borne diseases: Modeling through delay-differential equations, Dynamic Models of Infectious Diseases, Springer, 2013, pp. 43-75.

[25] Mariagrazia Mecoli, Vanda De Angelis, and Sally C Brailsford, Modelling the risk of mosquito-borne diseases by system dynamics: the case of human travel between different geographic regions, Systems Man and Cybernetics (SMC), 2010 IEEE International Conference on, IEEE, 2010, pp. 19-25.

[26] M Mimura and JD Murray, On a diffusive prey-predator model which exhibits patchiness, Journal of Theoretical Biology 75 (1978), no. 3, 249-262.

[27] Masayasu Mimura and Kohkichi Kawasaki, Spatial segregation in competitive interaction-diffusion equations, Journal of Mathematical Biology 9 (1980), no. 1, 49-64.

[28] Akira Okubo and Akira Okubo, Diffusion and ecological problems; mathematical models, Tech. report, 1980.

[29] WILLIAM RODERICK PLEMMONS, A mathematical study of malaria models of ross and ngwa, Ph.D. thesis, University of Central Florida Orlando, Florida, 2006. 
[30] Ronald Ross, An application of the theory of probabilities to the study of a priori pathometry. part $i$, Proceedings of the Royal Society of London. Series A 92 (1916), no. 638, 204-230.

[31] Natalia Sapoukhina, Yuri Tyutyunov, and Roger Arditi, The role of prey taxis in biological control: a spatial theoretical model, The American Naturalist 162 (2003), no. 1, 61-76.

[32] M. Singh, ALAN Easton, A., and I. Kozlova, A numerical study of the spruce budworm reaction-diffusion equation with hostile boundaries, Natural Resource Modeling 13 (2000), no. 4, 535-549.

[33] Gui-Quan Sun, Zhen Jin, Yi-Guo Zhao, Quan-Xing Liu, and Li Li, Spatial pattern in a predator-prey system with both self-and cross-diffusion, International Journal of Modern Physics C 20 (2009), no. 01, 71-84.

[34] J Tumwiine, JYT Mugisha, and LS Luboobi, A mathematical model for the dynamics of malaria in a human host and mosquito vector with temporary immunity, Applied Mathematics and Computation 189 (2007), no. 2, $1953-1965$.

[35] Pauline Van den Driessche and James Watmough, Reproduction numbers and sub-threshold endemic equilibria for compartmental models of disease transmission, Mathematical biosciences 180 (2002), no. 1, 29-48.

[36] Wendi Wang and Xiao-Qiang Zhao, A nonlocal and time-delayed reaction-diffusion model of dengue transmission, SIAM Journal on Applied Mathematics 71 (2011), no. 1, 147-168.

[37] Nicolay Nicolayevich Yanenko, The method of fractional steps, Springer, 1971. 